\title{
FORM OPTIMIZATION AND INTERACTION ANALYSIS OF PLANE SYMMETRY PRISM IN AIR
}

\author{
Shravan Koundinya Vutukuru, Igors Tipans, Janis Viba, Martins Irbe
}

Riga Technical University, Latvia

vshravankoundinya1989@gmail.com, igors.tipans@rtu.lv,janis.viba@rtu.lv, martins.irbe@rtu.lv

\begin{abstract}
In engineering, for many practical applications fluid-body interactions are commonly encountered and thereby these interactions have to be extensively studied. Though this body-fluid interaction is studied in the past, the concept of interaction analysis, form optimization relating to axis symmetry geometries till date mostly need to be further explored. The present work is devoted to the analysis of fluid (air) to body (prism) interaction, form (or shape) optimization taking into account various criteria and energy extraction from air flow by using an axial symmetric body (circular disc shaped geometry with alternate perforated quadrants) with plane symmetry (cylinders) is considered. A simplified mathematical model for engineering calculations is proposed. The model is based on the concept of pressure (compression) and suction zones, when a body is subjected to fluid (air) flow. Initially, all calculations are performed for the geometry under study in constant dimensions. Further, for better efficiency of the overall system, the system parameters are changed in constant continuous steps by a detailed analysis of the fluid-body interaction response surface. The mathematical model has the following assumptions: the fluid (air) flow is laminar, non-compressible (density constant) and the fluid (air) viscous is ignored. All the results obtained are discussed in graphs and explained. Three-dimensional fluid flow simulations are performed in ANSYS, steady state RANS is solved using $\mathrm{K} \varepsilon$ realizable turbulence model, the interaction force (Drag) is obtained for the geometry under study. Finally, the results relating to form (or shape) optimization and the application of the concept are widely discussed.
\end{abstract}

Keywords: flow-body interaction, energy generation, shape optimization.

\section{Introduction}

Fluid-rigid body interaction is an age-old, frequently occuring phenomenon, which finds wide application in engineering and medical fields when a rigid-body is completely immersed in fluid. Though this interaction phenomenon is well known, the analysis is not extended for the interaction force deviation( force along the flow direction) and further form optimization as a result of fluid-body interaction pertaining to axis symmetry geometries till date remains mostly unexplored.The basic idea behind the present work is to extend the concept of interaction analysis for estimating the interaction force deviation by varying the forms only in the leeward side (suction) as a simple task to optimize the interaction force and the same fluid - body interaction phenomenon is further extended to analyze the concept of energy extraction with the help of an alternate perforated circular disc in air through a straight forward mathematical model. It is realized that when the body is subjected to air flow, the space around the body in the fluid medium could be split into zones; upstream a pressure zone and down stream immediate to the body a suction or vacuum zone is formed [1-3]. In the present work the concept of zones is taken adavantage to analyse the task of form optimization by considering different stationary objects in the fluid flow. As understood from the existing research work that viscosity plays a secondary role in estimating the performance [4], the viscous effects are ignored, but the shape factors are still considered here in the present work due to the form of the prism (star geometry ) and system parameters are changed based on the fluid body interaction response.

In the present work, the form optimization task for a stationary body is analysed using ANSYS (3D Flow simulations) and an important conclusion has been drawn keeping in view the concave and convex object forms and further, importance of form optimization for excitation efficiency for the extraction of energy from the air stream is analysed in detail in later sections. All the equations are formed as per the laws of classical mechanics and the only disadvantage of the model is that the viscous nature of the fluid or surrounding medium is ignored when forming the mathematical model.

According to the laws of mechanics, for a stationary rigid-body fluid flow interaction, a wellknown practice is applied in the formula Eq. (1), [5]:

$$
F=\frac{1}{2} \rho V^{2} C_{d} A,
$$

where $F$-interaction force; 


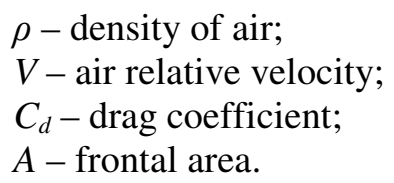

The drag coefficient $C_{d}$ is approximately constant in a region of high Reynolds number Re > 1000, Eq. (2) [5; 6]:

$$
\operatorname{Re}=\frac{\rho V l}{\mu},
$$

where $l$-characteristics length, the chord width of the object under study; $\mu$ - dynamic viscosity of the fluid.

In common engineering studies, when there is no change in the velocity $V$ both in magnitude and direction, the Reynolds number range (2) $(\operatorname{Re}>1000 ; v>0 ; \mu \rightarrow 0)$ permits the use of the formula (1).

In the case of non-stationary motion, the formula (1) cannot be used because of the constantly changing: the velocity of motion V, the coefficient $\mathrm{Cd}$ and the Reynolds number Re. Similarly, in the case of non - stationary motion, especially for the task of form optimization, the space - time programming method needs huge computational work and is time consuming. Therefore, it would be desirable for engineers to develop approximate methods that can be tested and applied to stationary motions, when: $V=$ const; $\operatorname{Re}=$ const; $C_{d}=$ const. For this purpose, the shape optimization problem model is shown in Fig. 1, the task here is to know the form of the prism (frontal, rear) section should be to obtain the minimum (or maximum) value of the criterion, if the prism is subjected to (immersed in) fluid flow.

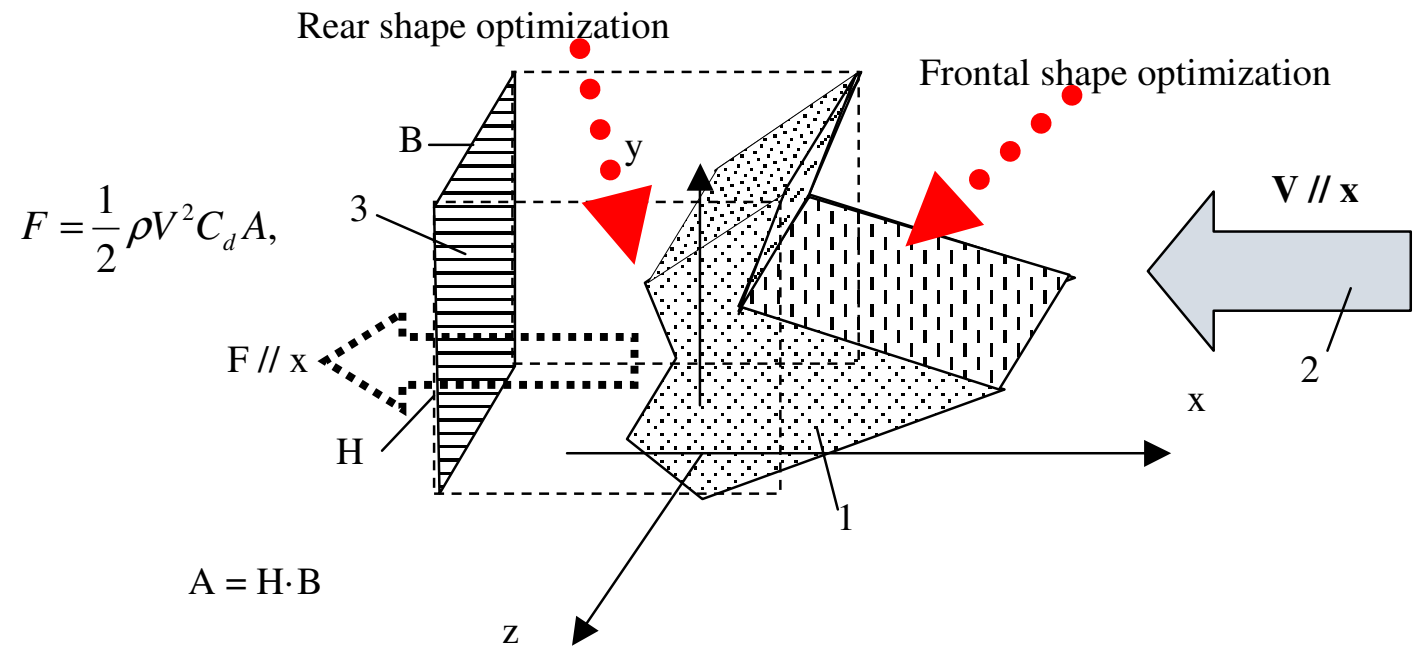

Fig. 1. Star prism stationary rigid body interaction with fluid flow: 1 - star shaped prism (cylinder); 2 - air flow with velocity V (from right to left); 3 - frontal area A, prism height B

Works related to special cases of non-stationary flow of objects are analytically described in [1-3]. In these works, the interaction is divided into the compression and suction zones for the space around the body in the fluid flow(air). It is explained in [1-3] that the suction side requires experimental or computer modeling studies to determine the interaction. Accordingly, on the compression (upstream) side, it is possible to calculate the interaction using classical mechanics: using the change in momentum. In the present work, at first we consider a stationary-body in flow, experimental studies of the drag force, here called as interaction force, values for various objects mentioned in Fig. 2 by looking at differently shaped intersecting objects and their interaction forces in fluid flow, important conclusions for all types of engineering calculations could be obtained, as mentioned in the following.

1. For a similar shape in the compression zone (upstream flow), but a different shape in the suction zone, the drag coefficient can be assumed constant for all engineering calculations (accuracy about till $2 \%$ for 2D model). 
2. The optimization criterion given here, when optimizing different shapes, should focus on the shape of the compression zone (most $C_{d}$ - dependent) or the interaction area A (for example, using perforated form).

3. It is important to note that for tasks that require the application of force in perpendicular to the flow (lifting force), the shape of the suction side must also be optimized (Fig. 1).

4. Thereby, the criteria for shape (form) optimization could be for any of the following : a) drag or lifting forces; b) vibration damping; c) excitation efficiency for the extraction of energy from the air stream (Fig. 1).
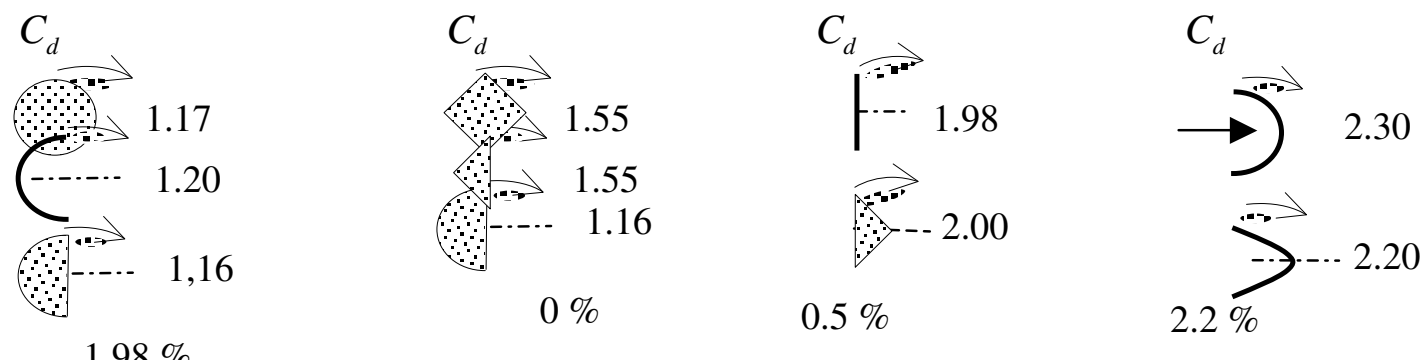

Fig. 2. Drag coefficient for axially prismatic 2 D flow [6]: $C_{d}-$ drag coefficient

Optimization challenges today are available in wide analytical theory, started in the middle of the 20 th century [7-10]. The main task is to solve the flow and object interaction, through approximate analytical relationships, which can be achieved as mentioned in the subsequent section. To this end, surfaces formed by fractured planes in the form of curved or concave geometric configurations, possibly with their perforations, are further discussed herein.

\section{Materials and methods}

\section{Model of convex broken side prism and analytical investigation method}

The convex broken side model four-corner prism is described in Fig. 3. For the frontal sides pressures $p 1, p 2, p 3$ the theorem of the change momentum of flow in the differential form can be applied [3] and the force can be calculated according to Eq. (3):

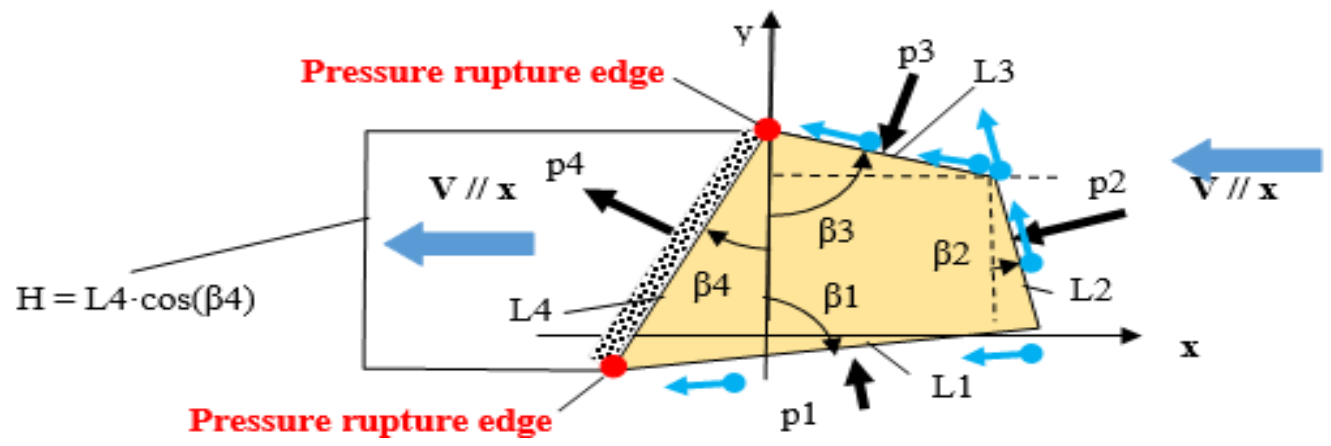

Fig. 3. Model of convex broken prism: $L 1, L 2$ - length of sides; $\beta 1, \beta 2$ - frontal angles; $H$ - height of the prizm; $\longleftrightarrow-$ symbol of flow direction on the body surface as a result of air to body interaction

$$
\begin{gathered}
p 1=[V \cdot \cos (\beta 1)]^{2} \cdot \rho ; p 2=[V \cdot \cos (\beta 2)]^{2} \cdot \rho ; \\
p 3=V^{2} \cdot \rho \cdot\left[\cos (\beta 3)^{2}-C 12 \cdot C 23 \cdot \cos (\beta 2) \cdot \sin (\beta 3-\beta 2)\right],
\end{gathered}
$$

where $\beta 1, \beta 2, \beta 3$ - orientation angles of the sides relative to the flow;

$C 12, C 23$ - constants that observe changes in the flow velocity along the flow line along the boundary layer at break points.

So, for example, $C 12=C 23=1$, means that the velocity at the point of the break does not change and is the same as for all the flow at the beginning, equal to $V$. Accordingly, the pressure $p 4$ in the suction zone between the edges of the rapture can be determined as follows Eq. (4) [1-3]: 


$$
p 4=C 4 \cdot V^{2} \cdot \rho
$$

where $C 4$ is a constant, for example $C 4=0.5$ [1].

It should be noted in the calculations that these formulas are applicable to prism that has convex surfaces in the pressure zone, for example:

$$
0<\beta 4<\pi \cdot 2^{-1} ; \pi>\beta 4+\beta 3>0 ; \beta 3-\beta 2>0 .
$$

Using equations (3) and (4), we can obtain the following projections of the interaction forces on the $\mathrm{x}$ and $\mathrm{y}$-axes (6), (7):

$$
\begin{aligned}
& \quad-F x=V^{2} \cdot B \cdot \rho \cdot\left\{L 1 \cdot[\cos (\beta 1)]^{3}+L 2 \cdot[\cos (\beta 2)]^{3}+\right. \\
& +L 3 \cdot\left[\cos (\beta 3)^{3}-C 12 \cdot C 23 \cdot \cos (\beta 2) \cdot \cos (\beta 3) \cdot \sin (\beta 3-\beta 2)\right]+ \\
& +L 4 \cdot C 4 \cdot \cos (\beta 4)\} ; \\
& -F y=V^{2} \cdot B \cdot \rho \cdot\left\{L 1 \cdot[\cos (\beta 1)]^{2} \cdot \sin (\beta 1)+L 2 \cdot[\cos (\beta 2)]^{2} \cdot \sin (\beta 2)+\right. \\
& +L 3 \cdot\left[\cos (\beta 3)^{2} \cdot \sin (\beta 3)-C 12 \cdot C 23 \cdot \cos (\beta 2) \cdot \sin (\beta 3) \cdot \sin (\beta 3-\beta 2)\right]+ \\
& +L 4 \cdot C 4 \cdot \sin (\beta 4)\} .
\end{aligned}
$$

When analyzing or optimizing these forces ( $F x$ - drag force (along flow direction), $F y$ - lift-force (perpendicular to flow direction) the following geometric links must be additionally observed (8), (9):

$$
\begin{aligned}
& L 4 \cdot \sin (\beta 4)+L 3 \cdot \sin (\beta 3)+L 2 \cdot \sin (\beta 2)-L 1 \cdot \sin (\beta 1)=0 ; \\
& L 4 \cdot \cos (\beta 4)-L 3 \cdot \cos (\beta 3)-L 2 \cdot \cos (\beta 2)-L 1 \cdot \cos (\beta 1)=0 .
\end{aligned}
$$

\section{Analytical investigation method of concave broken side prism}

In this case, there exists the impact force N3 on the edge of the inner breakage. The force N3 is perpendicular to the edge of the length L3, as shown in Fig. 4. According to boundary layer momentum exchange for the airflow, changing the direction from the edge L2 to edge L3, gives the impact force N3 (10):

$$
N 3=L 2 \cdot B \cdot \rho \cdot V^{2} \cdot \cos (\beta 2) \cdot \sin (\beta 2-\beta 3) \cdot[0.5+0.5 \cdot \operatorname{sign}(\beta 2-\beta 3)] .
$$

Here the criterion for taking concave case is when: $\sin (\beta 2-\beta 3) \geq 0$.

Accordingly, in the equations (6), (7) should be dropped the part by the minus sign, since this interaction is equivalent to $N 3$. However, if there is no such theoretical force in the flow interaction, the flow to be smooth over the entire surface with the length L3, it is recommended that the previous formulas (6), (7) are used in good approximation.

Thus, the relationships obtained above (8)-(10) allow for the analytical study of the interaction of various forms of star prisms, solving problems of optimization and synthesis.

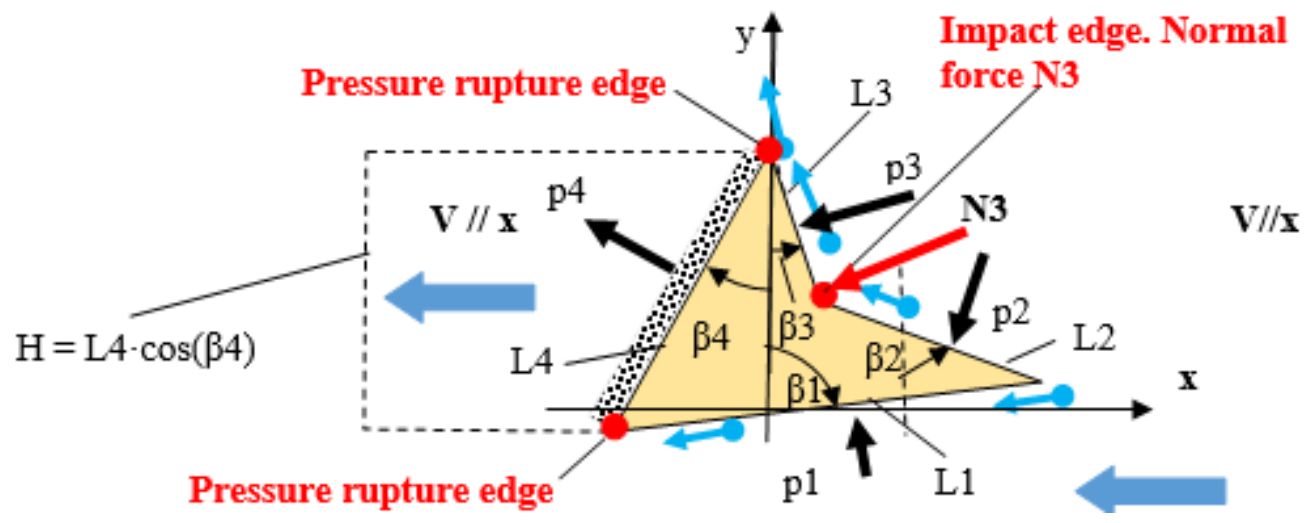

Fig. 4. Model of concave star like prism form: $L 1, L 2, L 3, L 4$ - length of sides; $\beta 1, \beta 2, \beta 3, \beta 4-$ frontal angles; $H$ - height of the prism 


\section{Example of form optimization of four - corner star prism}

In the parametric optimization problem, the horizontal force $F x$ (as a criterion) must be analyzed with the restrictions as mentioned, Eq. (8)-(10). Further given: the sides of the star are equal $L 2=L 3$, with the height $H=$ constant and $\beta 1=0$. Other parameters $V, \rho, B$ - remain constant. The results of optimization (maximum, minimum) $\mathrm{K}(\beta 2)=F x /\left(V^{2} \cdot \rho \cdot L 2\right)$ are shown in Fig. 5and Fig. 6 for different $C 12, C 23$ values. This example demonstrates that analytical relationships are obtained, which allow for parametric optimization of fluid flow interactions.

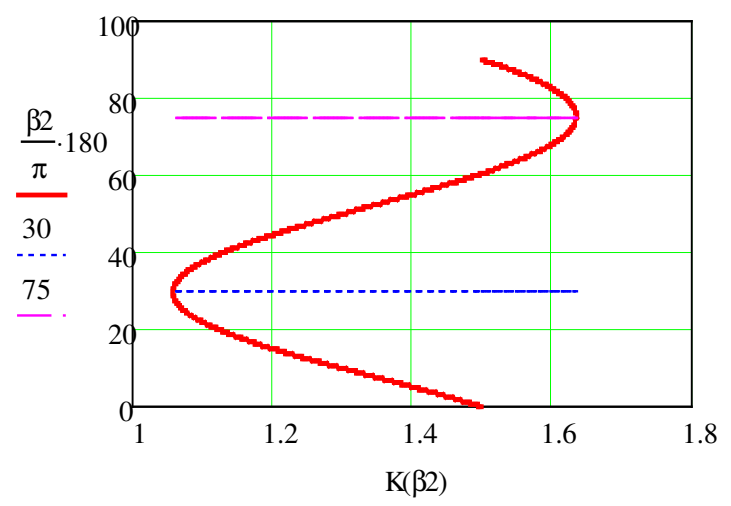

Fig. 5. Results of four sided -star prism optimization, first criterion: $K(\beta 2)-$ drag coefficient parallel to flow; $C 12=C 23=1$

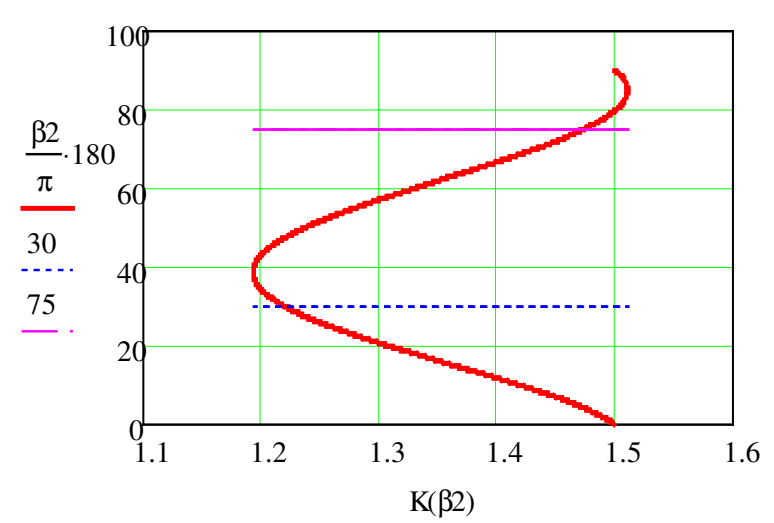

Fig. 6. Results of four sided - star prism optimization second criterion: $K(\beta 2)-$ drag coefficient parallel to flow; $C 12=C 23=0.5$

\section{Ansys 3D simulations}

In all 3D simulations performed in ANSYS , steady state RANS is solved using k- $\varepsilon$ realizable turbulence model. The total length of the domain was $4 \mathrm{~m}$, started at a length of $1.2 \mathrm{~m}$ from the body under consideration and the total height was $1.92 \mathrm{~m}$. A tetrahedron mesh with a total of 354221 elements and boundary layers set to stair stepping with maximum of 7 layers was used. The inlet condition specified as velocity inlet with a velocity of $10 \mathrm{~m} \cdot \mathrm{s}^{-1}$ and pressure outlet condition for rest of the domain sides. No slip condition was imposed at the stationary wall (geometry for all cases).

$B=0.16 \mathrm{~m} ; H=0.16 \mathrm{~m} ; \rho=1.225 \mathrm{~kg} \cdot \mathrm{m}^{-3}$ as shown in Fig. 7 , the interaction forces for the five forms of the prism were analyzed (Fig. 7). They differ from each other: a) three shapes at $90^{\circ}$ of uniform triangular at the pressure side (zone, upstream) with flat, or sharp spike $\left(20^{\circ}\right)$ or half-ridge (flow height equal to $H$ ) suction zone shapes; b) two shapes with the compression area at an angle of $20^{\circ}$ and a flat or sharp back form.

The main result of numerical modeling is that it confirms the above assumptions: in engineering calculations with sufficient accuracy (about $2 \%$ ), the pressure in the suction zone along the boundary layer is independent of the shape of the object. It is to be noted that the coefficients $C 12, C 23$ and $C 4$ were not analyzed in the present work.

\section{Parametric optimization of circular plate system}

An example of a flat circular plate with alternate perforated quadrants for energy extraction from the fluid flow [11] is shown in Fig. 8. Motion analysis in a 2D space is shown in Fig. 9-11. The system considered consists of two concentric circular plates fixed at the center, the plate which front area subject to fluid flow is to rotate freely over the other circular plate (non-rotating), both plates have the same area and alternate perforated quadrants, the spring, the linear generator in the incoming flow with a velocity $V 0\left(10 \mathrm{~m} \cdot \mathrm{s}^{-1}\right)$. 


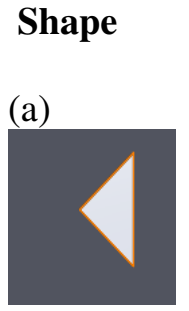

(a)

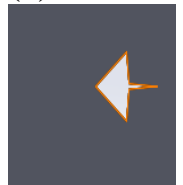

(a)

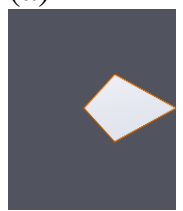

(b)

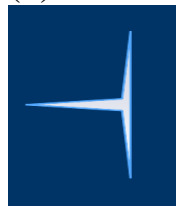

(b)

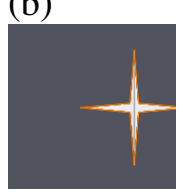

\section{Pressure contour}
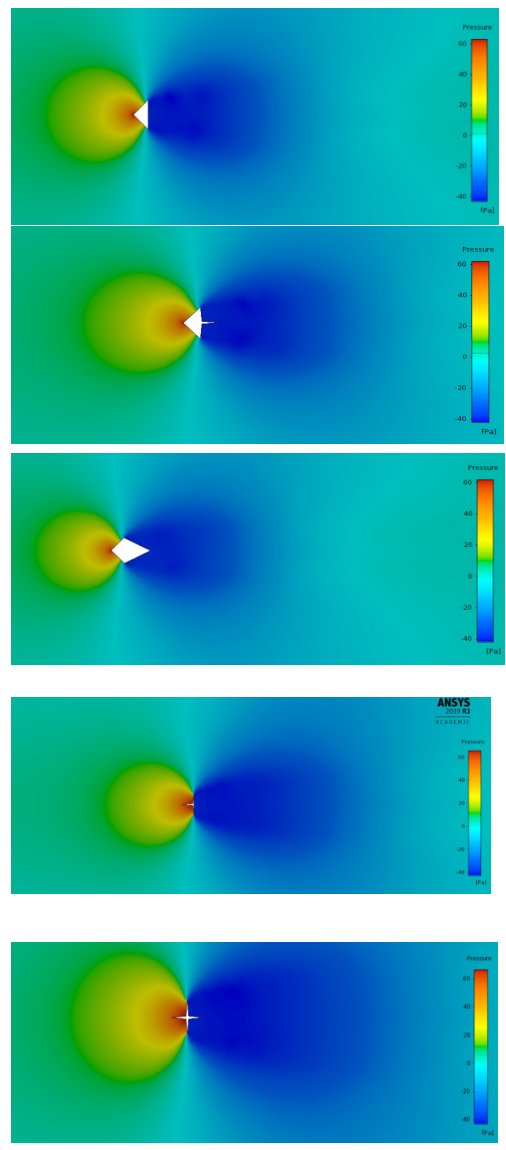

Streamlines
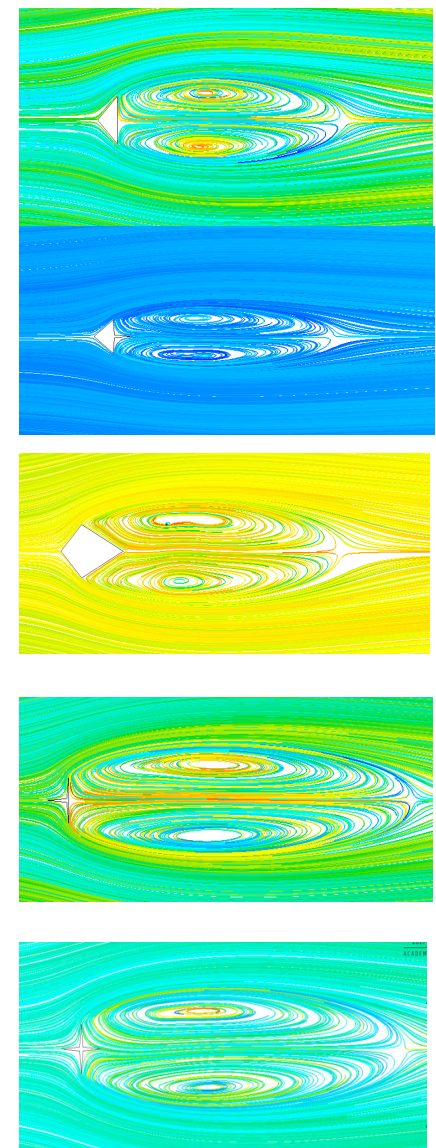

Axial force

deviation

$2.14 \%$

$0.15 \%$

$2.00 \%$

$0.10 \%$

$0.10 \%$

Fig. 7. Shape, pressure contour, streamlines for different shapes:

the percentage of mean deviation of the axial force

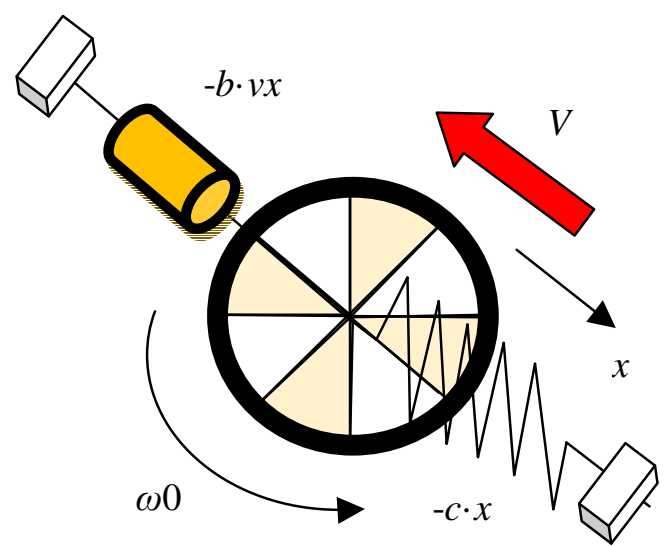

Fig. 8. Flat circular plate with alternate perforated quadrants: $V$ - flow velocity; $(-b \cdot v x)$

- generators force; $(-c \cdot x)$ - spring force; $\omega 0$ - angular velocity of rotating part

$$
\text { Control }=1+\frac{\arccos [\cos (\omega 0 \cdot t)]}{\pi}
$$

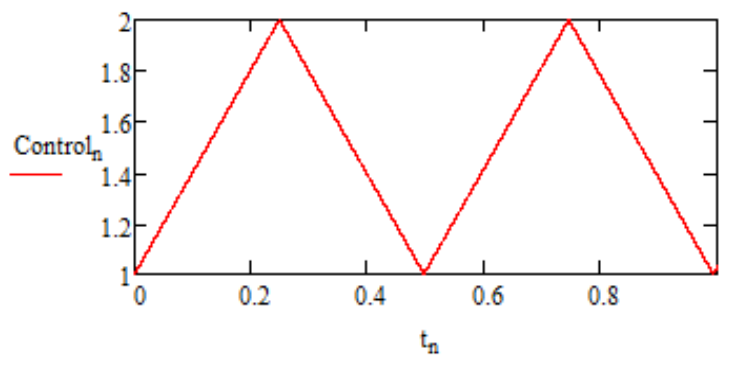

Fig. 9. Area control action: $t$ - time

Differential equation of plate motion is (11):

$$
\begin{aligned}
m \cdot \ddot{x} & =-c \cdot x-[F 0 \cdot \operatorname{sign}(\dot{x})-b \cdot \dot{x}]+ \\
& +(1+C) \cdot \frac{A}{\pi} \cdot[\operatorname{acos}[\cos (\omega 0 \cdot t)]+\pi] \cdot \rho \cdot(-V 0-\dot{x})^{2} \cdot \operatorname{sign}(-V 0-\dot{x}),
\end{aligned}
$$

where $m$-mass; 
$x$ - displacement;

$\dot{x}$ - velocity;

$c$-stiffness of the linear spring;

$F 0, b$ - constants of linear generator damping;

$(1+C)$ - coefficient of pressing and suction sides interactions;

$V 0$ - flow velocity;

$\omega 0$ - control actions harmonica angular frequency;

$A$ - constant area,

$\rho$ - air density, sign is +1 or -1 , depending of brackets signature.

The task of parametric optimization is as follows: find a combination within the seven-parameter ( $m, c, F 0, b, \omega 0, A, V 0)$ space with given constraints on the parameter values that provide the maximum power generated by the flow.

It is to be noted that the full resolution of such a task is not intended in this work. Note, however, that the best combination of the three parameters $(m, c, \omega 0)$ is close to the resonance zone:

$$
\omega 0=\sqrt{c \cdot m^{-1}} .
$$

The graphs for this case are shown in Fig. 10-11.

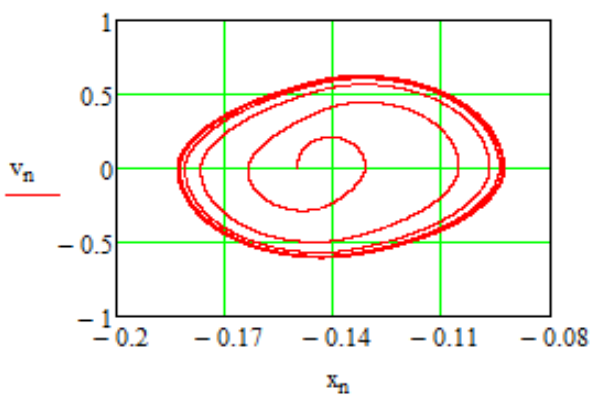

Fig. 10. Motion in phase plane:

$x$ - displacement of plate; $v$ - velocity of plate

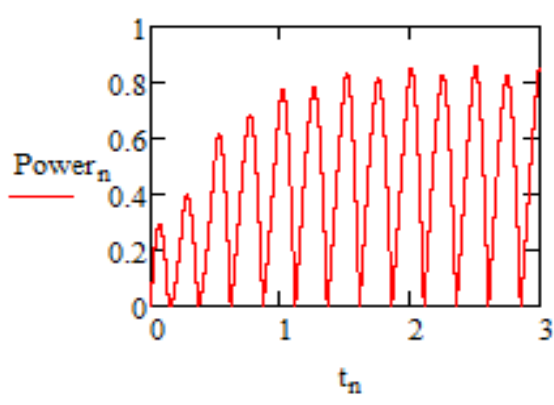

Fig. 11. Small plate power: $V 0$ - wind velocity $10 \mathrm{~m} \cdot \mathrm{s}^{-1} ; A-0.16 \mathrm{x} 0.16 \mathrm{~m} ; \omega 0=4 \cdot \pi, \mathrm{s}^{-1}$

\section{Results and discussion}

1. From the results obtained, it is confirmed that the calculations for the fluid body interaction task assume a constant pressure in the boundary layer of the flow suction zone at low speeds (air flow).

2. The pressure in the suction zone along the boundary layer is independent of the shape of the object, as it can be obtained from Fig. 7 above; this assumption is valid only at the low fluid flow speed.

3. Theoretically and by modeling with ANSYS, it is shown that there is a fundamental difference in the interaction forces between curved and concave object forms. It is shown that concave shapes should not be approximated by flat surfaces, as local forces are generated in the concave in the flow.

4. Form optimization criteria for different shapes involves the shape of the compression zone or the interaction area (when perforated geometries).

5. To obtain perpendicular interaction force to the flow (lifting force), the shape of the suction side (leeward side) should also be optimized.

6. A good fluid- body interaction analysis leads to analytical relations that can be extended for parametric optimization of fluid flow interactions.

7. Using the fluid - body interaction phenomenon, stable results are obtained for the energy extraction system in air that can be extended to any form in future.

\section{Conclusions}

1. This work further develops the analytical method previously proposed by the authors for the determination of fluid and object interaction forces in concave fractured forms. 
2. The proposed method can be extended to the study of non-stationary interactions that occur when the frontal area changes and when the relative flow velocity changes.

3. The method can be applied to solve technical problems of engineers in a simplified way, replacing work-space-time programming methods for bodies moving with low speeds.

\section{Acknowledgements}

This research is funded by the Latvian Council of Science, project "Creation of design of experiments and metamodeling methods for optimization of dynamics of multibody 3D systems interacting with bulk solids and fluids", project No. lzp-2018/2-0281.

\section{References}

[1] Tipans I., Viba J., Irbe M., \&Vutukuru S.K. Analysis of Non-Stationary flow interaction with simple form objects. Agronomy Research. Vol. 17(1), 2019, pp. 1227-1234.

[2] Vutukuru S.k., Viba J., Tipans I., Viksne I., and Irbe M. Analysis of flat Plate Vibrations by varying frontal area to flow. Engineering for rural development. 2019, pp. 1408-1414.

[3] Tipans I., Viba J., Vutukuru S.k. and Irbe M.Vibration analysis of perforated Plate in non stationary motion. Vibro-engineering PROCEDIA, vol. 25, 2019, pp. 48-53.

[4] Kinsey T. and Dumas G. Parametric study of an oscillating Airfoil in a power - Extraction regime. AIAA. vol. 46, No.6, 2008.

[5] Clancy L.J. Aerodynamics. New York, London: Publishing by Pitman, 1975. 610 p.

[6] Sighard.F. Hoerner, Fluid-Dynamic Drag. 1965.

[7] Pontryagin L.S., Boltyanski V.G., Gamkrelidze R.V., and Mishchenko E.F. Mathematical theory of optimal processes Fizmatgiz. Moscow, 1961. 391 p. (in Russian).

[8] Boltyanski V.G. Mathematical Methods of Optimal Control (in Russian), Izdat. "Nauka", Moscow, 1966. 307p. English translation: Holt, Rinehart and Winston, Inc., New York Montreal-London. Xiv, 1971, pp. 272.

[9] Lee E. B. and Markus L. Foundations of Optimal Control Theory. NewYork: Wiley.1967. 576 p.

[10] Boltyanski V., Martini H., and Soltan V. "The Maximum principle -How it came to be?" Geometric Methods and Optimization problems. New York: springer, 1998, pp. 204-227.

[11] Viba J. Optimisation and Syntheses of Vibroshock Machines. Riga, Zinatne. 1988, 253 p. (In Russian). 